\title{
Singular Covers in Free Lattices
}

\author{
RALPH FREESE and J. B. NATION* \\ Department of Mathematics, 2565 The Mall, Honolulu, HI 96822, U.S.A. \\ J. JEŽEK and V. SLAVÍK \\ MFF, Univerzity Karlovy, 18600 Praha 8, Sokolovská 83, Czechoslovakia
}

\section{Communicated by B. Jónsson}

(Received: 10 July 1985; accepted: 10 February 1986)

Abstract. A covering $a>b$ in a lattice is called a singular cover if $a$ is join irreducible and $b$ is meet irreducible. A classification of the singular covers which occur in free lattices is given.

AMS (MOS) subject classification (1980). 06B25.

Key words. Cover, free lattice, connected component.

\section{Introduction}

If $a$ covers $b$ (denoted $a>b$ ) in a lattice $L$, we call this a singular cover (or the pair $(a, b)$ a singular covering pair) if $a$ is join irreducible and $b$ is meet irreducible. This note characterizes such covers in free lattices. Whenever $a \prec b$ in a lattice $L$ then, by Dilworth's characterization of lattice congruences, there is a unique largest congruence $\psi(a, b)$ not containing $(a, b)$. Moreover in this situation $L / \psi(a, b)$ is subdirectly irreducible. When $L$ is a free lattice, these subdirectly irreducible lattices are always finite and are called splitting lattices. Such lattices were extensively studied by McKenzie [13]. Notice that if $(a, b)$ is a singular covering pair, then the congruence generated by $(a, b), \theta(a, b)$, identifies only $a$ and $b$. Thus if a subdirectly irreducible lattice has a singular covering pair $(a, b)$ then this is the only critical quotient. (A critical quotient in a subdirectly irreducible lattice is one that generates the unique minimal nontrivial congruence.) Many important splitting lattices have singular covers for their critical quotients. For example, the congruence lattices of the finitely generated free algebras in Polin's variety have this property [4]. Another such class of lattices is investigated in [3].

If $L=F L(X) / \psi(w, v)$, where $w>v$, is a splitting lattice with a singular cover, it does not follow that $(w, v)$ is a singular covering in $F L(X)$. However, the question of what are the singular coverings in $F L(X)$ is still interesting. In their study of covers in free lattices, Freese and Nation needed to understand singular covers in free lattices which satisfied some additional assumptions. Those authors were able to show that if $w \succ v$ is a singular cover satisfying these additional hypotheses, then either $v$ is the join of two

\footnotetext{
${ }^{\star}$ This research was supported by NSF Grants DMS83-18482 (Freese) and MCS83-01087 (Nation).
} 
atoms or $w$ is the meet of two coatoms. They asked if there were other singular covers in free lattices. Ježek and Slavík were able to prove for any singular cover in a free lattice there is a generator comparable to both the elements of the pair. Using this they were able to prove that the only singular covers in $F L(3)$ were those described above. Freese and Nation, using the results of [9] and [6], were then able to extend this result to all free lattices.

THEOREM. If $w>v$ is a singular cover in a free lattice, then either $v$ is the join of two distinct atoms or $w$ is the meet of two distinct coatoms.

In $F L$ (3) $x(x y+x z+y z) \succ x y+x z$ is indeed a singular covering pair. Likewise, if we let $a_{i}$ denote the atom $\Pi_{j \neq i} x_{j}$ of $F L(n),(n \geqslant 4)$, then for each $i, j$ with $1 \leqslant i<j \leqslant n$,

$$
\prod_{k \neq i, j}\left(a_{i}+a_{j}+a_{k}\right) \succ a_{i}+a_{j}
$$

is a singular covering pair in $F L(n)$. Of course, the duals of these are also singular covering pairs.

Our proof of the theorem will use quite a bit of the machinery developed in [9] and [6] for studying covers in free lattices. However, the argument for $F L(3)$ involves some interesting and different techniques, so we will sketch some of the important ideas here.

In [15] Whitman proved the free lattices satisfy the following continuity property. We write $a_{n} \uparrow a$ if $\left\{a_{n}: n \geqslant 0\right\}$ is an assending chain and $a$ is the least upper bound for this chain. Whitman showed that if $a_{n} \uparrow a$ in $F L(X)$ and $u \in F L(X)$ then $a_{n} \cdot u \uparrow a \cdot u$. From this it follows that if $a_{n} \uparrow a$ and $b_{n} \downarrow b, b \leqslant a$, and $b_{m} \nless a_{n}$ for all $m$ and $n$, then the interval $[b, a]$ contains no singular cover. Indeed, if $w \succ v$ were a singular cover in $[b, a]$, then $a_{n} \cdot w \uparrow a \cdot w=w$. If $a_{n} \cdot w<w$ then $a_{n} \cdot w \leqslant v$. Hence, for some $n$, $a_{n} \cdot w=w$, i.e., $w \leqslant a_{n}$. By duality $b_{m} \leqslant v$ for some $m$. Hence, $b_{m} \leqslant a_{n}$, a contradiction.

Let $a$ be the meet of the coatoms and $b$ the join of the atoms in $F L(X)$. Let $X=$ $\left\{x_{1}, \ldots, x_{n}\right\}$ and for $c \in F L(X)$ define

$$
f(c)=\prod_{i<j}\left(c \cdot x_{i}+c \cdot x_{j}\right)
$$

We define $g(c)$ dually. Let $b_{0}=a$ and let $b_{n+1}=f\left(b_{n}\right)$ and define $a_{n}$ dually. Then, with some work, one can show that $a, b, a_{n}, b_{n}, n \geqslant 0$, satisfy the hypothesis of the result of the previous paragraph. Hence $[b, a]$ contains no singular cover. Notice that $[b, a]$ is precisely the set of all elements which are not comparable with a generator. With the aid of this and some additional work one can show that the only singular covers in $F L(3)$ are those described in the theorem.

\section{Preliminaries}

Before we begin the proof of the theorem we will need to review some basic facts about free lattices and their covers. In [10] Whitman solved the word problem for free lattices. We assume the reader is familiar with his solution. Part of his solution is the condition (W): 
(W)

$$
\begin{aligned}
& v_{1} \ldots v_{n}=v \leqslant u=u_{1}+\cdots+u_{m} \text { implies } \\
& v_{i} \leqslant u \text { for some } i \text { or } v \leqslant u_{j} \text { for some } j
\end{aligned}
$$

which holds in $F L(X)$. Note this implies that every element of $F L(X)$ is either meet or join irreducible. We say that $\left\{w_{1}, \ldots, w_{k}\right\}$ refines $\left\{u_{1}, \ldots, u_{m}\right\}$, and we write $\left\{w_{1}, \ldots\right.$, $\left.w_{k}\right\} \ll\left\{u_{1}, \ldots, u_{m}\right\}$, if for each $i$ there is a $j$ such that $w_{i} \leqslant u_{j}$. Whitman also showed that for each $w \in F L(X)$ there is a term of minimal length representing $w$, unique up to commutativity and associativity. If $w=w_{1}+\cdots+w_{k}$ is this representation, where each $w_{i}$ is not formally a join, and if $w=u_{1}+\cdots+u_{m}$, then $\left\{w_{1}, \ldots, w_{k}\right\} \leqslant\left\{u_{1}, \ldots, u_{m}\right\}$. We call $\left\{w_{1}, \ldots, w_{k}\right\}$ the canonical joinands of $w$. It follows from this that if $a<b$ in $F L(X)$ then there is a unique largest element $c$ with $c \geqslant a$ but $c \neq b$. Namely, $c$ is the unique canonical meetand of $a$ which is not above $b$. In particular, if $w$ is completely join irreducible, i.e., $w$ is join irreducible and has a unique lower cover (always denoted) $w_{*}$, then there is a unique largest element, denoted $k(w)$, satisfying $k(w) \geqslant w_{*}$ and $\kappa(w) \neq w$, and $\kappa(w)$ is the unique canonical meetand of $w_{*}$ which is not above $w$. If $q$ is completely meet irreducible, we let $\kappa^{\prime}(q)$ be the map dual to $\kappa$. Note $\kappa$ and $\kappa^{\prime}$ are inverses of each other.

Another consequence of this refinement property of canonical joins is the fact that $F L(X)$ satisfies Jónsson's semidistributive laws, $\left(\mathrm{SD}_{\vee}\right)$ and $\left(\mathrm{SD}_{\wedge}\right)$ :

$$
\left(\mathrm{SD}_{\vee}\right) a+b=a+c \text { implies } a+b=a+b \cdot c \text {. }
$$

If $w$ is a join, say $w=\Sigma w_{i}$, canonically, then the following lemma shows that the lower covers of $w$ are determined by the completely join irreducible canonical joinands of $w$.

LEMMA 1.1 (Corollary 2.4 [9]). Let $w=\Sigma w_{i}$ canonically in $F L(X)$. If $w_{i}$ is completely join irreducible then $w>w \cdot \kappa\left(w_{i}\right)$. Conversely, if $w>v$ and $w_{i}$ is the unique joinand not below $v$, then $w_{i}$ is completely join irreducible and $w_{i *}=v \cdot w_{i}$. Moreover in this case every element below $w$ is either below $v$ or above $w_{i}$.

We will need several technical theorems about covers in free lattices from [9]. If $w$ has the form

$$
w=\prod_{i}\left(\sum_{j} w_{i j}\right) \prod_{k} x_{k}
$$

where the $x_{k}$ 's are generators, then $J(w)$ is defined recursively to be $\{w\}$ if $w$ is a meet of variables, and

$$
\{w\} \cup \bigcup_{i, j} J\left(w_{i j}\right)
$$

otherwise. The set $M(w)$, for $w$ meet irreducible, is defined dually.

THEOREM 1.2 (Theorem 4.3 [9]). Let $w$ be a join irreducible element of $F L(X), X$ finite, and let

$$
w_{\dagger}=\sum\{u \in J(w): u<w\} \text { and } K(w)=\left\{v \in J(w): w_{\dagger}+v \ngtr w\right\} .
$$


Then $w$ is completely join irreducible if and only if

(i) every $u \in J(w)-\{w\}$ is completely join irreducible, and

(ii) $w \nless \Sigma K(w)$.

Notice that in particular if $w$ is completely join irreducible then so is every element of $J(w)$.

THEOREM 1.3 (Theorem 4.4 [9]). If $w$ is a completely join irreducible element of $F L(X)$ and has the canonical form (1) where each $x_{k} \in X$, then for each $i$ there is exactly one $j$ with $w_{i j} \nless w$.

By rearranging elements we can always assume that $j=1$, so that we always have $w_{i 1} \nless w$.

THEOREM 1.4 (Theorem 4.7 [9]). If $w$ is completely join irreducible then the canonical meetands of $w_{*}$ are $\{k(w)\} \cup\left\{w_{i}: w_{i} \neq k(w)\right\}$.

The next theorem gives a useful characterization of three element intervals in free lattices.

THEOREM 1.5 (Theorem 10.2 [9]). Suppose $w$ is join irreducible and the middle element of a three element interval $w_{*} \prec w \prec u$. Then $w \notin X, u$ is join reducible, and if $q$ is the canonical meetand of $w$ not above $u$ then $w=q \cdot u$ canonically and $q$ is completely meet irreducible.

If we define an equivalence relation $\sim$ by $a \sim b$ if there is a sequence $a=a_{0}, a_{1}, \ldots$, $a_{n}=b$ such $a_{i}$ covers or is covered by $a_{i+1}$, then the connected components (of the covering relation) of the free lattices are the equivalence classes of this relation, thought of as subpartially ordered sets of the free lattice A complete description of all these connected components is given in [6]. We present those parts of this description that we require below.

For $n \geqslant 4$ the connected component of the least element, $0=\Pi x_{i}$, of $F L(n)$ consists of 0 , the $n$ atoms, their $\left(\begin{array}{l}n \\ 2\end{array}\right)$ pairwise joins, and $\left(\begin{array}{l}n \\ 2\end{array}\right)$ elcments covering each of these pairwise joins. This component, for $n=4$, is diagrammed in Figure 1. The connected component for $n=3$ is also given by the diagram below. In both diagrams the singular covers are labelled. In the first, $a_{i}=\Pi_{j \neq i} x_{j}$ is an atom.

Note that the join of any two atoms of a free lattice is the lower element of a singular pair.

\section{The Proof}

Now we are ready to begin the proof of the theorem. We begin with two lemmas. From now on we shall assume that $w>v$ is a singular cover in $F L(X)$ and that the canonical form of $w$ is given by (1), where, as before, $x_{k} \in X$ and for each $i, w_{i 1} \nless v$. 

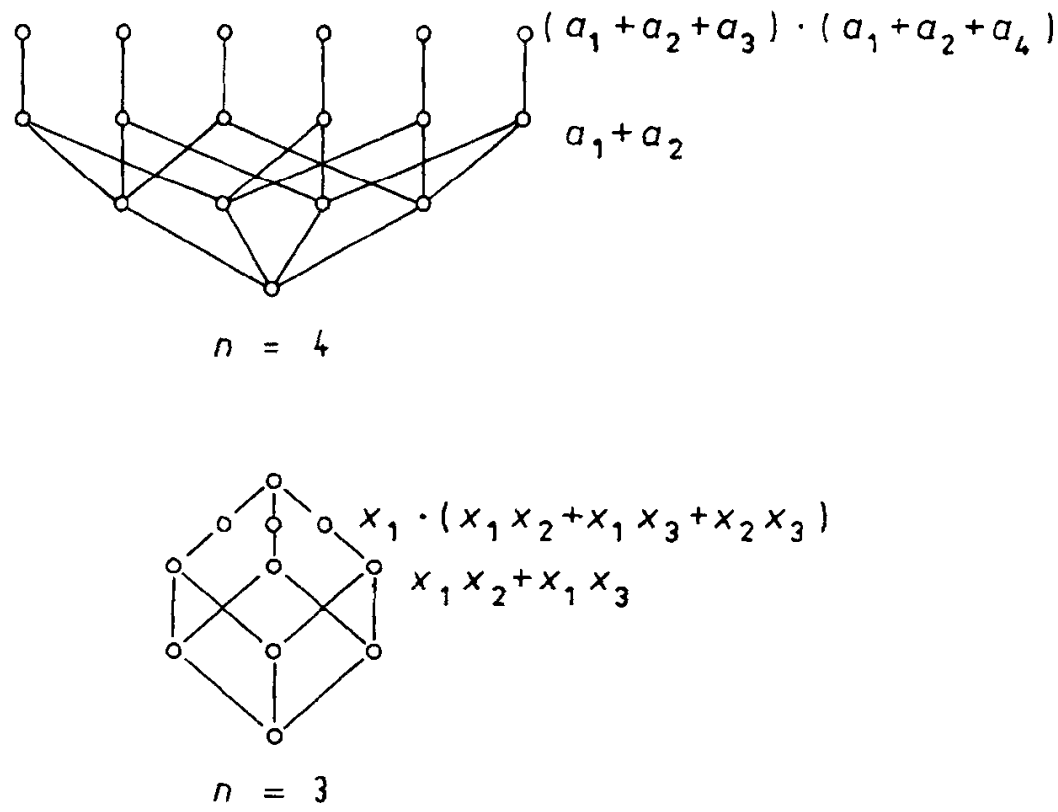

Fig. 1.

LEMMA 2.1. For all $i, w_{i 1 *} \leqslant v$.

Proof. Suppose that for some $i, w_{i 1 *} \$ v$. Then since $v^{*}=w$ we have

$$
\prod_{i} w_{i} \cdot \prod_{k} x_{k}=w \leqslant w_{i 1 *}+v
$$

By $(W)$ this implies that for some $j, w_{j} \leqslant w_{i 1 *}+v$, or for some $k, x_{k} \leqslant w_{i 1 *}+v$. Since $w_{i 1 *}+v \leqslant w_{i}$, the first case must hold with $j=i$, and thus $w_{i 1 *}+v=w_{i}$. By the refinement property of the canonical joinands described in the last section $\left\{w_{i 1}, w_{i 2}, \ldots\right\} \ll$ $\left\{w_{i 1 *}, v\right\}$. However, $w_{i 1} \nless w_{i 1}$ and $w_{i 1} \nless v$. This contradiction proves the lemma.

LEMMA 2.2. Either $w$ has an upper cover or $v$ has a lower cover.

Proof. Suppose not. Then, by Lemma 1.1, no generator is a canonical meetand of $w$, i.e., $w=\Pi_{i} \Sigma_{j} w_{i j}$, and dually $v=\Sigma_{r} \Pi_{s} v_{r s}$.

Since, by Theorem 1.3, all the canonical joinands of $w_{i}$, except $w_{i 1}$, lie below $w$, $w_{i}=w_{i 1}+v=w_{i 1}+\Sigma v_{r}$. It follows that

$$
\left\{w_{i 2}, w_{i 3}, \ldots\right\} \ll\left\{v_{1}, v_{2}, \ldots\right\}
$$

for all $i$.

For any pair $i, r$ we have

$$
\prod_{s} v_{r s}=v_{r} \leqslant w_{i}=\sum_{j} w_{i j}
$$

We wish to apply (W) to this inclusion. Suppose $v_{r} \leqslant w_{i j}$ for some $j>1$. By $(*) w_{i j} \leqslant v_{t}$ 
for some $t$, whence $r=t$ and $v_{r}=w_{i j} \in J(w)$. Thus, by Theorem 1.2, $v_{r}$ is completely join irreducible and $v>v \cdot k\left(v_{r}\right)$, contrary to our assumption. Hence $v_{r} \nless w_{i j}$ for $j>1$, and dually $v_{r s} \$ w_{i}$ for $s>1$. We conclude by (W) that for any pair $i, r$ either $v_{r 1} \leqslant w_{i}$ or $v_{r} \leqslant w_{i 1}$.

Assume that $v_{r} \leqslant w_{i 1}$. Then $v_{r} \neq w_{i 1}$, since $v_{r} \leqslant w$, so $v_{r} \leqslant w_{i 1 *}$. On the other hand, $w_{i 1 *} \leqslant v$, by Lemma 2.1. Hence, $w \cdot w_{i 1}=w_{i 1 *} \leqslant v=\Sigma_{t} v_{t}$. Applying (W), we find that $w_{i 1 *} \leqslant v_{t}$ for some $t$. It follows that $t=r$ and $w_{i 1 *}=v_{r}$. By duality, if $v_{r 1} \leqslant$ $w_{i}$ then $v_{r 1}{ }^{*}=w_{i}$. Thus, we have that for any pair $i, r$ either $v_{r 1} *=w_{i}$ or $w_{i 1} *=v_{r}$.

Now let $i=1$ and let $r$ vary. Clearly $w_{11 *}=v_{r}$ for at most one $r$, so there exists $t_{1}$ such that $v_{r 1}{ }^{*}=w_{1}$ for $r \neq t_{1}$. Likewise, there exists $t_{2}$ such that $v_{r 1}{ }^{*}=w_{2}$ for $r \neq t_{2}$. Since $w_{1} \neq w_{2}$, it follows that there are only two $v_{r}$ 's, i.e., $v=v_{1}+v_{2}$ canonically.

From the case $i=1, r=1$ we get either (a) $v_{11}{ }^{*}=w_{1}$ or (b) $v_{1}=w_{11 *}$. From $i=1$, $r=2$ we get either (c) $v_{21} *=w_{1}$ or (d) $v_{2}=w_{11 *}$, and from $i=2, r=1$ either (e) $v_{11} *=$ $w_{2}$ or (f) $v_{1}=w_{21 *}$. It follows that either (a) and (f) hold, or else (b) and (c) hold. By duality, we may assume the latter, i.e., $v_{1}=w_{11 *}$ and $v_{21} *=w_{1}$.

Note that $w_{11} \leqslant v_{21}$. For otherwise, (b) would imply $v_{1} \leqslant w \cdot w_{11} \leqslant w \cdot v_{21}=v_{2}$, a contradiction.

Now consider $w_{1}=w+w_{11}=w+v_{21}$ (by (c)). By $\left(\operatorname{SD}_{\vee}\right)$ this implies $w_{1}=w+$ $w_{11} \cdot v_{21}=w$ since $w_{11} \nless v_{21}$ and $w_{11 *} \leqslant v \leqslant w$. This final contradiction proves the lemma.

Returning to the proof of the Theorem, we again assume that $w \succ v$ is a singular cover. By Lemma 2.2 and duality we may assume that there is an element $u$ covering $w$. Since $v$ is meet irreducible, the interval $u / v$ is a three element chain. By Theorem 1.5 there is a completely meet irreducible element $q$ such that $w=u \cdot q$ canonically. Moreover, if $u_{1}$ denotes the unique canonical joinand of $u$ not below $w$, then $u_{1}=\kappa^{\prime}(q)$. This situation is diagrammed in Figure 2. The solid lines are used to indicate coverings.

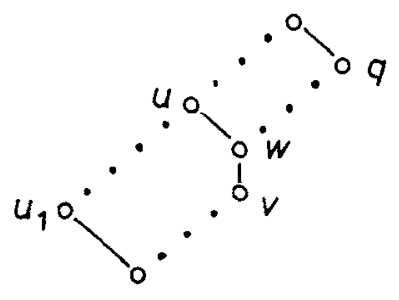

Fig. 2.

Now $u=u_{1}+v=u_{1}+\Sigma v_{r}$. Since $u=\Sigma u_{i}$ canonically we obtain from the refinement property of Section 2 :

$$
\left\{u_{i}: i>1\right\} \ll\left\{v_{1}, v_{2}, \ldots\right\} .
$$

Note that there is an $r$ such that $v_{r} \notin u_{1}$. For otherwise we would have $v \leqslant u_{1} \leqslant u$, which, since $u_{1} \nless w$, implies $u_{1}=u$. Since $u_{1}$ is a canonical joinand of $u, u=u_{1}$ implies 
that $u$ is either a generator of a proper meet. This however contradicts Theorem 1.5 which says that $u$ is join reducible.

Fix an $r$ such that $v_{r} \nless u_{1}$. Then $\Pi_{s} v_{r s}=v_{r} \leqslant u=\Sigma u_{i}$. Applying $(W)$ to this inclusion we get four cases.

Case 1. $v_{r} \leqslant u_{1}$. This cannot occur by the choice of $r$.

Case 2. $v_{r} \leqslant u_{i}$ for some $i>1$. By $(* *)$ we also have $u_{i} \leqslant v_{s}$ for some $s$, whence $r=s$ and $v_{r}=u_{i} \in J(w)$. Since $w$ is completely join irreducible, $v_{r}$ must be completely join irreducible by Theorem 1.2, and so $v \quad v \cdot \kappa\left(v_{r}\right)$ by Lemma 1.1. Hence $u \succ w>v>$ $v \cdot k\left(v_{r}\right)$ is a covering chain of length 3. By Theorem 9.2 of [9], such chains occur only in the connected component of $F L(X)$ containing 0 or 1 , where the known singular covers in $F L(X)$ also occur. Thus we may assume that $v_{r} \$ u_{i}$ for all $i>1$.

Case 3. $v_{r s} \leqslant u$ for some $s>1$. Now $v_{r s}>v$ (as $s>1$ ) and $v_{r s}$ is meet irreducible, so this implies $v_{r s}=u$. But $v_{r s} \in M(v)$ is completely meet irreducible, i.e., $u=v_{r s}$ has an upper cover $u^{*}$. Again this gives a covering chain of length three, so we may assume that this case does not occur.

Case 4. $v_{r 1} \leqslant u$. By the dual of Lemma 2.1 we have $v_{r 1} * \geqslant w$, so $w \leqslant v_{r 1} * \leqslant u$. This implies that $v_{r 1}{ }^{*}=u$, since $v_{r 1}^{*}=w$ would imply that $w=v_{r 1}+v$ is a proper join, a contradiction.

Let $u_{2}$ be the canonical joinand of $u$ not below $v_{r 1}$, so that $v_{r 1}=u \cdot \kappa\left(u_{2}\right)$. Now $v_{r 1}$, being a canonical meetand of $v_{r}$, is meet irreducible, so in fact $v_{r 1}=\kappa\left(u_{2}\right)$. We claim that $u_{1} \neq u_{2}$. Clearly $v_{r 1} \neq w$ since $w$ is meet reducible and $v_{r 1}$ is meet irreducible. Also $v_{r 1} \nless v$ by the definition of canonical form. Since every element properly below $w$ is below $v$, the above implies that $v_{r 1} \leqslant w$. Now if $v_{r 1} \leqslant q$ then $v_{r 1} \leqslant q \cdot u=w$, a contradiction. Thus $k\left(u_{2}\right)=v_{r 1} \notin q$, but $\kappa\left(u_{1}\right)=q$, showing that $u_{1} \neq u_{2}$.

Now apply the dual of Theorem 1.4. Since $u=v_{r 1}{ }^{*}$, the canonical joinands of $u$ are precisely $u_{2}$ and the canonical joinands of $v_{r 1}$ not below $\kappa^{\prime}\left(v_{r 1}\right)=u_{2}$. Thus each $u_{i}, i \neq 2$, and especially $u_{1}$, is a canonical joinand of $v_{r 1}$.

Hence,

$$
v_{r 1} \succ v_{r 1} \cdot k\left(u_{1}\right)=v_{r 1} \cdot q=v_{r 1} \cdot u \cdot q=v_{r 1} \cdot w=v_{r} .
$$

Since $v_{r} \leqslant v$, we have shown that the connected component of $u$ in $F L(X)$ contains at least the configuration diagrammed in Figure 3, where solid lines denote coverings.

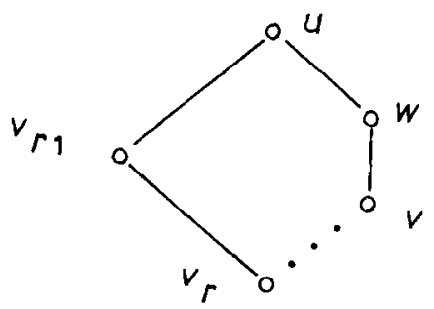

Fig. 3.

By checking the main theorem of [6], which characterizes connected components in 
free lattices, we see that the above configuration is never part of a connected component.

This final contradiction completes the proof of the theorem.

\section{References}

1. P. Crawley and F. P. Dilworth (1973) Algebraic Theory of Lattices, Prentice-Hall, Englewood Cliffs, NJ.

2. A. Day (1977) Splitting lattices generate all lattices, Algebra Universalis, 7, 163-169.

3. A. Day (1975) Splitting lattices and congruence modularity, Colloq. Math. Soc. Janos Bolyai, 17, Contributions to Universal Algebra, Szeged, 57-71.

4. A. Day and R. Freese (1980) A characterization of identities implying congruence modularity I, Can. J. Math. 32, 1140-1167.

5. R. Freese (1982) Some order theoretic questions about free lattices and free modular lattices, in Ordered Sets (ed. I. Rival), D. Reidel, Dordrecht, pp. 355-377.

6. R. Freese (1985) Connected components of the covering relation in free lattices, in Universal Algebra and Lattice Theory (ed. S. Comer), Lecture Notes in Mathematics, vol. 1149, SpringerVerlag, New York, pp. 82-93.

7. R. Freese, Free lattice algorithms.

8. R. Freese and J. B. Nation (1978) Projective lattices, Pacific J. Math. 75, 93 - 106.

9. R. Freese and J. B. Nation (1985) Covers in free lattices, Trans. Amer. Math. Soc. 288, $1-42$.

10. G. Grätzer (1978) General Lattice Theory, Academic Press, New York.

11. B. Jónsson (1982) Varieties of lattices: Some open problems, Colloq. Math. Soc. Janos Bolyai, 29, Contributions to Universal Algebra (Esztergom), North Holland, Amsterdam, pp. 421-436.

12. B. Jónsson and J. B. Nation (1977) A report on sublattices of a free lattice, Colloq. Math. Soc. Janos Bolyai, 17, Contributions to Universal Algebra (Szeged), North Holland, Amsterdam, pp. $223-257$.

13. R. McKenzie (1972) Equational bases and nonmodular lattice varieties, Trans Amer. Math. Soc. 174, 1 - 43.

14. P. Whitman (1941) Free lattices, Ann. Math. 42, 325-330.

15. P. Whitman (1942) Free lattices II, Ann. Math. 43, 104-115. 